\title{
TOWARDS “INVENTIVENESS-ORIENTED” CAI TOOLS
}

\author{
Denis CAVALLUCCI ${ }^{1}$, Noel Leon ${ }^{2}$ \\ ${ }^{1}$ Associate professor, INSA, Strasbourg; ${ }^{2}$ Full Professor, ITESM, Mexico
}

Abstract The emergence of a new category of tools baptised CAI (Computer Aided Innovation) is taking shape as industrial demand becomes more pressing. One category of tools in particular stands out as being a break from the usual trends in CAD, i.e. where the software structure is partially inspired by TRIZ. While the aim of such tools is to assist the designer in his creative act upstream of the act of designing, the link between the list of concepts these tools offer and the input required to build an object model is not ensured to date. The aim of this article is to clarify the essential factors characterising an act of designing/redesigning inspired by the fundamental aspects of TRIZ. This should help bridge the gap between the traditional knowledge (see Yoshikawa H., 1989) which contributed to the development of current CAD tools and the approach which makes TRIZ a theory totally in line with current trends in search of efficient inventiveness in terms of the design act.

Key words: inventive design, creativity, CAI, TRIZ, design

\section{INTRODUCTION}

Technical artefacts are becoming increasingly sophisticated as technology evolves. This logic of obeying needs, which are either latent or clearly expressed, is also evident in man's ever more impatient demands. At the same time, the buying power of households is increasing and the average cost of accessing innovations available on the market is falling. The result is that consumption of new products is continuously growing and this has a major impact on industry, where the need to rebuild design potential is strongly felt both in terms of human skills and methodological expertise. 
A new situation is therefore arising, affecting the designers of these artefacts. It can be summed up as follows: are the tools and methods developed to aid the designer in his tasks still appropriate in the context briefly outlined above?

Two fundamental aspects make us think this is not the case:

1. The gap between the rate of requests for human creativity and its actual capacity;

2. The gap between the scope of knowledge required in view of the level of complexity, and the inherent ability of a collective human group within a given organisation.

Contemporary designers are faced with a two-fold dilemma - that of having to ensure design tasks in a context where:

- The tools and methods available to assist them were developed within a context of optimising quality, as imposed in the 1960s1990s. This means they are not always adapted to meet the requirements of current design tasks which are more focussed on optimising creative potential (Shaw, M.C., 1986) leading to higher efficiency in terms of inventiveness in the design act within the company (Holtj K., 1992);

- The complexity of the artefact and the scope of knowledge required make their own creative capacity inadequate. This limitation is accentuated by the fact that a truly inventive act is often measured by the following yardstick: external knowledge (i.e. unknown at that time by the industrial sector in which the designer works) is technologically transferred to the designer's own field, thereby making the creative act inventive.

\section{THE LIMITATIONS OF THE DIVERGENT APPROACHES}

In this section, we would like to demonstrate that the current approaches used to deal with problems, which we shall call the divergent approaches, have the single aim of increasing the output of ideas statistically speaking. This type of approach is adopted with the aim of formalising a direction for the designing process, starting with a situation expressed as the initial problem (which is often vague or stems from a need which is more or less clearly expressed).

The creative phases situated upstream of this type of process will basically be conducted with the aim of finding a maximum number of 
ideas to make up a sufficient statistical population. This is then followed by a multi-stage sorting process to isolate the idea which best matches the initial specifications. Such creative acts are mainly based on brainstorming or similar sessions, or consulting databases to scan any solutions that either already exist at rival companies or are in the process of development in research laboratories. The sorting process following the creative phases are either a simple filter weeding out ideas deemed too far-fetched or eliminating ideas which cannot integrate the data given in the initial specifications. The outcome of the sorting process is to limit the number of ideas to the most relevant and to set them in a hierarchy so as to have alternative development plans which more or less break with the current state of knowledge in the company. It is then up to the decision-makers to select whichever alternative matches their own strategy.

This type of process has two obvious limitations:

- The direction chosen is necessarily induced by ideas expressed during creativity sessions and therefore depends on a random process of exploiting the knowledge of the people involved and cannot guarantee at any moment that the direction chosen is truly the optimum direction.

- The exhaustive collection of ideas depends solely on the skills and knowledge of the persons who take part in the creativity sessions. This means it is impossible to guarantee that the statistical spectrum of ideas expressed embodies the ideal solution to the problem posed.

We shall therefore summarise this section with the following postulate: adopting a divergent design process does not guarantee that the directions selected for the designing process are ideal and therefore, the costs generated through iteration on the basis of the unsatisfactory results obtained (whether it is through prototyping and tests, calculations or $R \& D$ ) place the company in a trial-and-error context, which is costly for the inherent added value, as it generates significant costs in terms of man-hours.

The challenge for designers of tools and methods aimed at assisting artefact designers is therefore clear for the years to come: to enable designers to optimise their creative potential (Mars N.J.and al., 1993) through means other than brainstorming and similar processes or accessing catalogues of typical solutions in a given field.

Within the context of making CAI tools, our starting point will be the definition of the ideality of such a system in terms of TRIZ (Altshuller G.S., 1986, 1988). 
The tool assisting the physical design of artefacts must enable the designer to optimise the inventive aspect of his approach by instantly giving an accurate profile of the optimum model solution for the problem posed using all the initial data.

Among existing solutions, some of which are partially based on theoretical aspects of TRIZ (see Tsourikov V., 1993), we observe that in spite of the fact that they encompass many of the aspects linked to TRIZ databases and tools, the philosophy of how the problem is dealt with differs little from the divergent approach. The next section of our paper therefore aims to clarify the foundations required for a CAI tool to be made, by associating it with an input element and interacting with a traditional CAD architecture. Such experiments have already been carried out by the company Dassault Systems in the "Product Function Optimizer 2 (PFO)" option in version 5 of their CAD tool CATIA, but our contribution aims here to reposition this type of development in line with what makes sense in TRIZ: the convergent process.

\section{TOWARDS MAKING INVENTIVENESS- ORIENTED COMPUTER TOOLS}

\subsection{The generic model characterising a CAD approach}

The model given in figure 1 summarises the generic treatment process for a societal need for technical system design requiring a CAD tool. It should be noted that this type of model follows the divergent logic described above.

\subsection{The generic model proposed in our paper}

First of all, we shall attempt to base the main lines of our reflections on the theoretical approach to inventiveness-seeking as developed by Genrich Altshuller. Contrary to the divergent approach described in the previous section, this theory is based on the postulate that it is necessary to reduce the scope of research by putting a halt to investigations in fields and sectors which are irrelevant because the goals in question, the specific conditions dictated by the context and the development trends of technical systems do not indicate that this is the most logical direction to turn to for the development of the artefact. On the other hand, the picture of the solution is built up step by step by adding, as the theory unfolds (which may be associated with a method), all the elements necessary to build the profile of 
the solution. We are therefore adopting a guided design approach (and not random design) and an improvement can be noted compared to the two stumbling blocks apparent in the divergent approach.

- The direction selected is defined in keeping with the laws of technical system evolution. These laws have been developed over a period of nearly half a century following observations of the evolution of a large quantity of technical artefacts throughout their history. They have been validated by pertinent scientific analyses (Salamatov Yu.P., 1991) and regular iterations and are now presented in a synthetic form enabling the essential points of the evolutionary dynamics of a technical system to be fully understood.

- The exhaustiveness of the fields investigated is also enhanced since Altshuller's theory encompasses a set of databases which synthesise human inventive mechanisms used by inventors in all industrial sectors. These databases are models of the cognitive, technical and scientific knowledge of human inventive activity synthesised in a semantic form that can be exploited in creative contexts (as also expressed in Akman V., 1989).

Finally, the experience provided by the practical application of this theory leads us to conclude that the robustness of the outcome depends on two essential factors - the relevance of the analysis carried out and a sound knowledge and grasp of the basics highlighted by Altshuller. Designing computing products inspired by the approaches advocated by TRIZ should therefore be in keeping with the following basic points. These basic points may also be considered as a system of factors enabling the profile of the designing process to be built: 


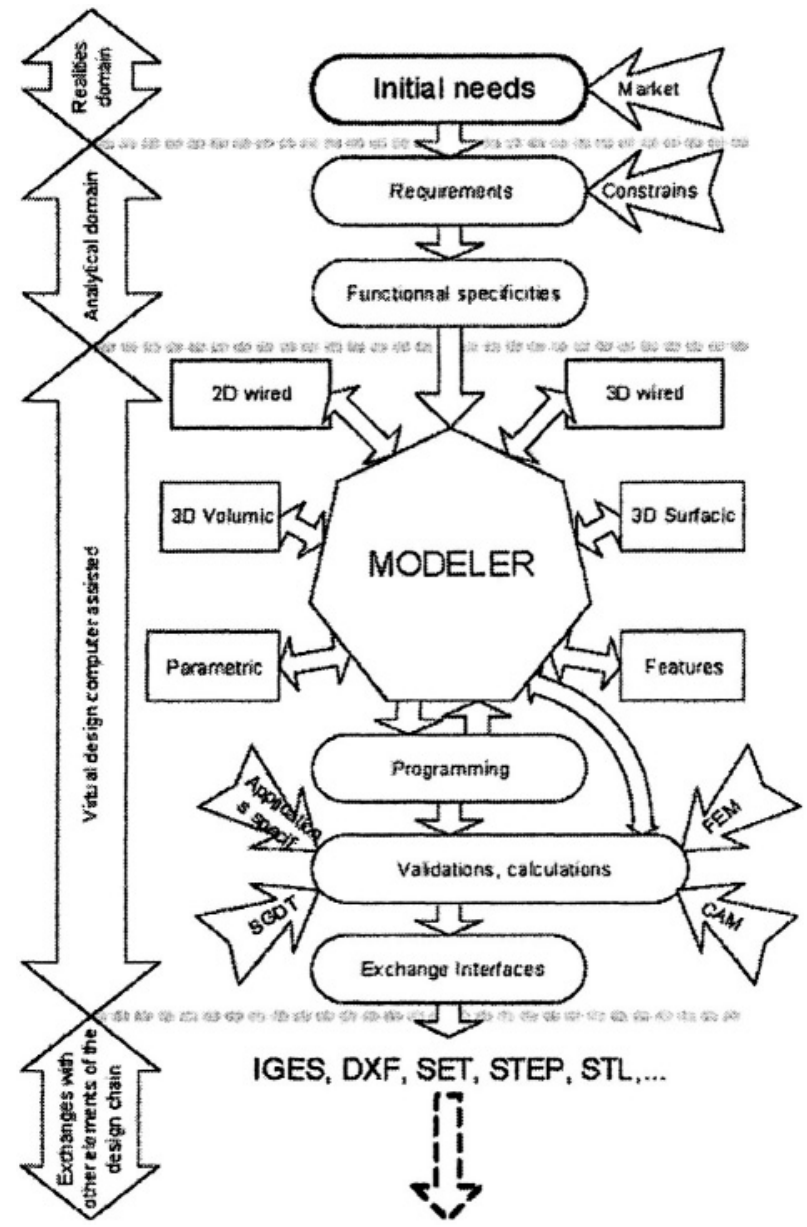

Figure 1. Generic model representing a traditional CAD approach

Definition of a final ideal objective: as the ultimate phase in the evolution of a designed artefact. In TRIZ, it is defined as minimising the material and energy resources required for its primary useful function, while maximising the functions it takes on for man. We should note that the ultimate phase is attained when the artefact becomes immaterial and its functions are automatically carried out.

- Example for CAD: Automatically designing an artefact on the basis that the alternative suggested to the designer goes in the direction of minimising the number of parts, favouring convergence and maximising the opportunities of creating new functions without increasing the level of complexity. 
Relevant list of resources: They are indicated in TRIZ as being the key elements in the problem-solving process. By definition, a resource may be a substance, a physical field, a space or a time. The resources which should be focussed on are in the operating zone and are present during the operating period of producing the primary useful function.

- Example for CAD: Benefiting at all times from a balanced list of resource items available (functional surfaces, physical fields present, spaces between objects, operating times calculated by simulation, etc.) during the entire period of design so I can refer to it while systematically designing my virtual model.

Designing up a contradiction network: All design acts are carried out as cognitive acts encouraging the designer to solve a contradiction introduced by his act. This essential notion in TRIZ stipulates that the contradiction symbolises the obstacle which has to be understood and solved to enable the technical system to evolve in keeping with the laws (as expressed in Killanders A. \& V. Sushkov, 1995). While cognitive reflexes often drive designers to a compromise solution, Altshuller purports that compromise does not arise from an inventive approach and that to move in the direction of inventiveness, the designer must refuse compromise despite his psychological inertia to solve the dilemma posed by the contradiction. The level of complexity involved in designing an artefact implies that a network of contradictions should be built up in order to place the designer face to face with the challenges he has to raise.

- Example for CAD: Constantly having a visual element which states contradictions of type: "The complexity of my shape must be reduced to facilitate its machinability yet significant to simplify its integration in my virtual unit". These contradictions are then collected in a coherent network modelling the complex situation the designer has to deal with.

Exploiting this network to initiate the design path: The contradiction network helps the designer to build a model of the problem in order to reduce its complexity. A set of guiding factors must then be designed for this network to enable the designer's problem-solving actions (or possibly his choices) to be directed towards an inventive approach, bearing in mind the company's strategy problems.

- Example for CAD: Having an iteration factor in the contradiction network which means links and their hierarchy can be visualised again. A contradiction may take on a different form if the objective behind the design act evolves from a desire to reduce costs to a desire to favour the inventive nature of the potential solution. 
The factor ofpin-pointing concordance between the directions taken and the laws: Beyond the brief introduction to the laws in the previous paragraph. As a tool for assisting the design act, the laws can constitute cartographic factors enabling the designer to situate himself in the action logic. Any approach to using a computing tool such as CAI must therefore be confronted with its coherence in relation to the laws of evolution so as to guarantee the relevance of the directions taken and possibly put the designer face to face with alternatives which may allow his action to be in line with the company's strategy.

- Example for CAD: The subordinate position of the approach compared to the laws of technical system evolution must offer the designer an overall view of his act and its impact on the evolution of the artefact. To do this, the generic breakdown of each of the laws (solid link, hinge, several hinges, flexible link, liquid link, field type link etc.) must offer the designer a choice of alternatives in which the current position of his system is located and the decision-making approach he must adopt may be conducted in keeping with the breakdown of the laws.

Access to databases and their graphic form: An important deliverable in terms of the computing tool features is also to provide access modes to TRIZ-model databases that are coherent with the typology of the designer's problem model. Links must therefore be designed between the graphic model representing the contradiction network and the databases representing inventive reflexes which inventors experienced in similar problem patterns (not moving towards compromise by definition).

Example for CAD: During the decision-making phases of contradiction-solving, the alternatives proposed by TRIZ databases will have evolved in terms of their formulation since they are associated with additional items collected during the analysis phase. These alternatives must all constitute a bolster for the thought process enabling the designer to structure his inventive response to the contradiction set by his problem model.

\subsection{Method of representing a system (from the TRIZ angle)}

The representational method we will use stems from Altshuller's first law of evolution: the law of the wholeness (completeness) of parts. This law stipulates that for a system to ensure its primary useful function, it must have four basic parts ideally fulfilling their role in their respective functioning so 
that by bringing them together we have an additional function (the Primary Useful Function) and not merely a simple combination of their respective functions.

These four major parts are:

- the driving element:

The function of which is to recover the energy required to make the system come up to expectations and to transform it into an energy which can ensure the PUF.

- the transmission element: Which, through its very structure, will carry this energy towards the working factor while attempting to optimise the notion of transfer efficiency.

- the working element: Which, within the system under study, will ensure the contact between our system and the factor on which it is supposed to deliver the PUF.

- the control element: Of which the primary function is to interact and react to variations in the system's functioning by playing the role of an "orchestra conductor" by guiding one or more of the three factors quoted above (through a modification to form, structure, properties and informational output).

A graphic representation of law 1 is given in Figure 2. Its purpose is to specify the interrelations between the parts of the system and it is also used to give the physical outline of the system under study. 




Figure 2. Representative model of the elementary architecture of a system from the TRIZ viewpoint, translated as a model for CAI

\subsection{Suggested generic model}

All the factors of the "CAI" system must form a coherent whole helping the designer to formalise his technical problem and come up with an inventive solution (at this stage, still in a theoretical form) on a basis which idealises the technical solution to be built. This coherence must also be in line with the corporate strategy (desire to offer new products, to promote one's position as market leader, to make profits by minimising the risks linked to innovation etc.).

\subsection{Proposal for an inventive design model}

The component parts of the diagram represented in Figure 3 may take the following shape so as to be integrated into the model presented in section 1 :

The means of fulfilling the technical specifications for each of the entities described in the model undeniably remains to be worked on in an efficient partnership between researchers in the field of TRIZ and researchers designing fundamental structures for CAD tools. We firmly believe that the fundamentals should spring from the fruit of this cooperation leading to a relevant and long-lasting emergence of a new generation of tools that we may then entitle CAI as they will match the requirements relating to innovation in industrial environments. 


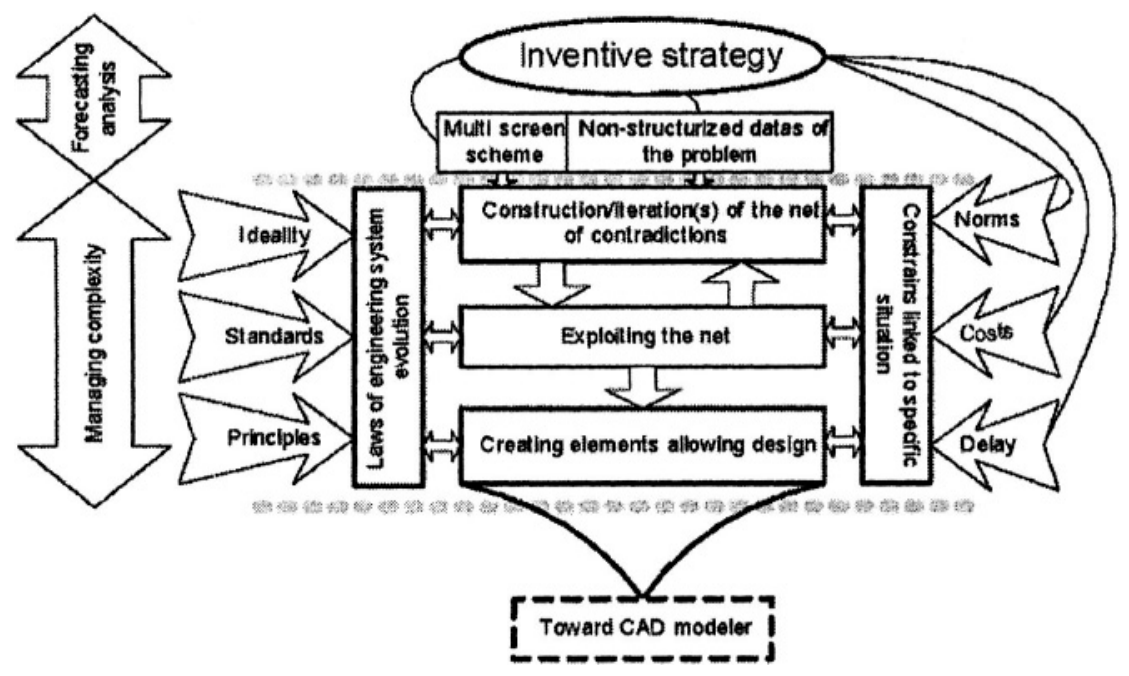

Figure 3. Proposal for a CAI model architecture

\section{CONCLUSIONS}

In this, our contribution aimed at designers of computing tools related to artefact-designing, we argue that the traditional reflex to move towards the compromise solution must be overcome in the search for inventive design. This design may be called inventive if it pursues an ultimate ideal objective through a dynamic process based on laws which characterise the evolution of technical systems. An essential component of these laws stipulates that a technical artefact evolves when it solves one of its contradictions, helped as far as possible by the resources present in the operating zone where the conflict between the contradictory aspects occur. Altshuller and his associates followed the aim of developing this theory for more than half a century, iterating its means decade after decade in a broad spectrum of tools and methods, teachings and algorithmisation. Today, this theory, which is emerging in our Western society, is the first to uphold the creative act of the designer by combining a synthesis of knowledge collected from human inventive activity and an objective guide to the design act based on characterising what is inventive and what is not (Cavallucci, D., 1999).

It should also be noted that our contribution is no more than a rough tree structure of the "what". In other terms, what are the key factors in a project 
leading to an inventive design approach? The expected and logical follow-up is now to investigate the "how" - firstly making the fundamentals coherent and then laying down links with the act of designing the artefact in a relevant way, which CAD tools already manage to do fairly well. Finally, the efficient diffusion of such tools can only occur once a large stumbling block has been overcome - that of providing a pedagogical structure so that it can be assimilated by designers whose professional reflexes have been deadened by their inertia towards the divergent approach, which is still firmly rooted anchored in our Western educational systems.

\section{REFERENCES}

1. Akman V., 1989, “The power of Physical Representations", AI Magazine, pp. 49-65. Altshuller G.S., 1986, "To Find an Idea: Introduction into the Theory of Inventive Problem Solving”, Nauka, Novosibirsk (in Russian).

2. Altshuller G.S., 1988, "Creativity As An Exact Science", Gordon \& Breach, New York, USA, ISBN 0-677-21230-5.

3. Cavallucci, D., 1999, "Contribution à la conception de nouveaux systèmes mécaniques par intégration méthodologique", Doctoral Thesis, Université Louis Pasteur, Strasbourg, France.

4. Dixon J.R., 1987, “On Research Methodology Towards a Scientific Theory of Engineering Design”, Affificial Intelligence for Engineering Design, Analysis and Manufacturing, Vol. 1, No. 3, pp. 145-157.

5. Holtj K., 1992, “Computer- aided creativity in engineering design”, Journal of Engineering Design, 4(4), 371-376

5. Jones J.C., 1981, "Design methods: seeds of human futures", Wiley and Son, New York. Killanders A. \& V. Sushkov, 1995, "Conflict-Oriented Model of Creative Design", in Proceedings of the 3rd Int. Roundtable Conference on Computational Models of Creative Design, Queensland, Australia, 369-397.

6. Mars N.J., P.M. Wognum \& V.V. Sushkov, 1993," AI and Creative Design", in Artificial Intelligence \& Creativity Papers from the 1993 Spring Symposium, T. Dartnall \& S. Kim, eds., Stanford, AAAI Press, 51-56.

7. Salamatov Yu.P., 1991, "A System of Laws of Engineering Evolution", in Chance for Adventure, A.B. Selutsky (ed.), Petrozavodsk, Karelia, 5-174 (in Russian).

8. Shaw, M.C., 1986, "Creative Design," CIRP Annals 35(2):461-465.

9. Tsourikov V., 1993, “Inventive Machine: second Generation”, AI and Society, Vol. 7, $\mathrm{N}^{\circ} 1$, Springler International, UK, 1993.

10. Yoshikawa H., 1989, "Design Philosophy: The State of the Art", Annals of the CIRP, vol. $38 / 2$. 